\title{
es protéines de la famille pRb sont des intégrateurs des signaux antiprolifératifs
}

Les trois membres de la famille $\mathrm{pRb}$ ( $p 107, p 130$ et pRb, ce dernier étant le produit du gène de susceptibilité au Rétinoblastome Rb1) sont des régulateurs transcriptionnels essentiels du contrôle intrinsèque de la prolifération cellulaire chez tous les eucaryotes supérieurs. Les travaux décrivant l'invalidation d'un ou de deux de ces gènes chez la souris ont révélé une importante redondance fonctionnelle (ou une possibilité de compensation fonctionnelle) entre les différents membres de cette famille (Tableau I). Afin d'évaluer la fonction de la famille $\mathrm{pRb}$ dans son ensemble, deux équipes dirigées respectivement par les $\mathrm{Dr}$ H ein te Riele (NKI, Amsterdam, Pays-Bas) et Tyler Jacks (MIT, Cambridge, USA) viennent de créer par recombinaison homologue des cellules de souris ES (embryonic stem cells) et MEF (mouse embryo fibroblasts) n'exprimant plus aucune de ces protéines (cellules TKO pour triple knock out $\mathrm{Rb}^{-1-} x$ p107-I- $x$ p130 $10^{-1-}$. . L'analyse phénotypique de ces cellules est décrite dans deux articles publiés dans le numéro de décembre 2000 de la revue Genes and Development $[1,2]$. Cette analyse confirme sans ambiguité le rôle essentiel joué collectivement par ces protéines dans le contrôle négatif de la prolifération.

\section{Les protéines de la famille pRb}

Les protéines de la famille $\mathrm{pRb}$ sont des phosphoprotéines nucléaires qui forment des complexes multiprotéiques avec plusieurs facteurs de transcription liant I'ADN. Les plus abondants et les mieux caractérisés de ces complexes sont ceux formés avec les facteurs de transcription de la famille E2F. Ces complexes E2F jouent un double rôle au niveau des promoteurs sur lesquels ils se fixent : un rôle de répresseur actif lorsqu'ils sont associés à une protéine de la famille $p R b$, et un rôle de transactivateur en leur absence. Les gènes contrôlés (de façon positive ou négative) par ces complexes E2F/ pRb codent pour des enzymes, facteurs de transcription et régulateurs divers, dont les fonctions sont essentielles pour la progression de la phase G1 vers la phase $\mathrm{S}$ du cycle cellulaire. II s'agit de régulateurs du cycle et de la croissance cellulaire, d'enzymes, de régulateurs de la biosynthèse de I'ADN, ainsi que de l'apoptose (voir revue dans [3]). L'expression transitoire de ces gènes est contrôlée par le cycle d'association et de dissociation des complexes E2F/ pRb, qui est luimême réglé via la phosphorylation des protéines des familles $p R b$ et E2F par les cyclines/ cdk de la phase G1 puis $S$ du cycle cellulaire (cycD1/ cdk4-6, puis cycE/ cdk2 et cycA/ cdk2). De nombreux travaux montrent que cette cascade constitue un intégrateur pour de multiples signaux cellulaires agissant sur la prolifération: signaux positifs (facteurs de croissance...) ou négatifs (cytokines du type TGF $\beta$, lésions de I'ADN, privation en sérum, confluence, sénescence, différenciation). Cette double fonction de gardien de la transition G1/ S du cycle cellulaire et d'intégrateur de l'état physiologique de la cellule, fait de cette cascade une cible du processus de transformation cellulaire. De fait, son fonctionnement est fréquemment (toujours selon certains auteurs) déréglé dans les tumeurs humaines. Ainsi, les gènes INK $4 a$ (inhibiteurs des cdk4, 6) et RB1 sont des suppresseurs de tumeur avérés et Cycline D1, D2, cdk4 et E2F, des oncogènes (voir revue dans [3-5]).

\section{Redondance fonctionnelle et spécificité des protéines de la famille $\mathbf{p R b}$}

D'une façon générale, l'étude expérimentale de cette cascade de régulation est rendue difficile par l'implication, à chacun de ses niveaux, de familles de protéines dont les membres sont soit fonctionnellement et naturellement redondants, soit capables de compenser partiellement l'absence de l'un des leurs. Ce propos est parfaitement illustré par les protéines de la famille $\mathrm{pRb}$. Les analyses phénotypiques des souris $\mathrm{R}^{-1-}$, p107- et $p 130^{-/-}$, ainsi que celle des doubles mutantes $\mathrm{pR}^{-1 / /} \mathrm{p} 107^{-1-}$, p10 $7^{-1 /} / \mathrm{p} 30^{-1-}$, se sont révélées peu informatives, suggérant à la fois la présence d'une forte redondance fonctionnelle et l'existence de quelques fonctions spécifiques, encore mal définies, dont l'absence aboutie à des défauts de développement tardifs (tableau I) (voir revue dans [4]).

Plus décevante encore, I'analyse de fibroblastes embryonnaires en culture issus de ces souris, simples ou doubles mutantes, montre que ces cellules demeurent capables de répondre partiellement à la plupart des signaux d'arrêt de prolifération, suggérant à nouveau la présence d'une forte redondance/ compensation fonctionnelle entre les différents membres de la famille (T ableau I).

\section{Cellules triple knock out $\mathrm{Rb}^{-/-} \times \mathrm{p} 107^{-/-} \times \mathrm{p}^{130^{-/-}}$}

Une évaluation du rôle global joué par les protéines de la famille $\mathrm{pRb}$ dans le contrôle de cette cascade est enfin disponible grâce à la création de cellules ES mutantes pour les trois membres de la famille du gène $R b[1$, 
Tableau I. Conséquences de l'inactivation des gènes codant pour les protéines de la famille pRb.

\begin{tabular}{|c|c|c|c|c|}
\hline Souris & Phénotype des souris & Réf. & Phénotype des MEF & Réf. \\
\hline $\mathbf{R b}^{-/+}$ & - Tumeurs de l'hypophyse et de la thyroïde & 6,7 & -Aucun phénotype particulier & 8,9 \\
\hline $\mathbf{R b}^{-/-}$ & $\begin{array}{l}\text { - Létalité embryonnaire }(\mathrm{E} 13,5, \mathrm{E} 15,5) \\
\text { - Défaut de l'érythropoïèse } \\
\text { - Apoptose dans système nerveux central, } \\
\text { foie, rétine }\end{array}$ & 6,7 & $\begin{array}{l}\text { - Phase G1 plus courte } \\
\text { - Modification de l'expression de } \\
\text { certains régulateurs du cycle cellulaire } \\
\text { - Faible capacité à se différencier in vitro } \\
\text { (fibroblastes } 3 T 3 \mathrm{Rb}^{-/} \text {) }\end{array}$ & $-10,12$ \\
\hline p130--- & $\begin{array}{l}\text { C57BL/6: } \\
\text { aucun phénotype particulier } \\
\text { Balb/C: } \\
\text { - Létalité embryonnaire (E11, 13) } \\
\text { - Retard de croissance } \\
\text { - Apoptose dans SNC et muscle }\end{array}$ & 15 & -Aucun phénotype particulier & $\begin{array}{l}9,11 \\
12\end{array}$ \\
\hline p107-- & $\begin{array}{l}\text { C57BL/6: } \\
\text { aucun phénotype } \\
\text { Balb/C } \\
\text { - Syndrome myéloprolifératif } \\
\text { - Hyperplasie de la rate et du foie }\end{array}$ & $\begin{array}{r}11,13 \\
14\end{array}$ & $\begin{array}{l}\text { C57BL/6: } \\
\text { aucun phénotype particulier } \\
\text { Balb/C } \\
\text { - Temps de doublement plus rapide }\end{array}$ & $\begin{array}{l}9,11 \\
14\end{array}$ \\
\hline $\begin{array}{l}\text { p130-/- } \\
\text { p } \\
\text { p07-/- }\end{array}$ & $\begin{array}{l}\text { - Létalité néonatale (insuffisance respiratoire) } \\
\text { - Malformations osseuses et des cartilages }\end{array}$ & 11 & $\begin{array}{l}\text { - Modifications de l'expression de certains } \\
\text { gènes réglés au cours du cycle cellulaire } \\
\text { - Phase G1 plus courte et phase } S \text { plus longue } \\
\text { - Forte capacité à se différencier in vitro } \\
\text { (fibroblastes 3T3) }\end{array}$ & $9-12$ \\
\hline $\begin{array}{l}\mathbf{R b}^{-/-} \\
\mathbf{x} \\
\mathbf{p} 107^{-/-}\end{array}$ & $\begin{array}{l}\text { - Létalité embryonnaire }(\mathrm{E} 15,5)^{*} \\
\text { - Des animaux chimériques viables } \mathrm{Rb}^{-/-p 107^{--}} \\
\text {développent des rétinoblastomes }\end{array}$ & $\begin{array}{l}13 \\
16\end{array}$ & $\begin{array}{l}\text { - Modifications de l'expression de certains gènes } \\
\text { réglés au cours du cycle cellulaire } \\
\text { - Phase G1 plus courte et phase S plus longue } \\
\text { - Perte partielle de la capacité à s'arrêter en } \\
\text { réponse aux signaux antiprolifératifs }\end{array}$ & $\begin{array}{c}1,9 \\
13,16\end{array}$ \\
\hline $\begin{array}{l}\mathrm{Rb}^{-/-} \\
\text {p107-/- } \\
\text { x } \\
\text { p130-/- }\end{array}$ & Non réalisées & & $\begin{array}{l}\text { - Cellules immortalisées } \\
\text { - Prolifération plus rapide, phase G1 plus courte } \\
\text { - Pas d'arrêt en G1 en réponse à divers signaux } \\
\text { antiprolifératifs (stress génotoxiques, inhibition } \\
\text { de contact, privation en sérum, croissance à } \\
\text { basse densité, sénescence) } \\
\text { - Croissance en agar mou } \\
\text { - Ne forment pas de tumeurs chez les souris Nude }\end{array}$ & 1,2 \\
\hline
\end{tabular}

2]. Ces cellules ont été obtenues par plusieurs étapes successives de recombinaison homologue. Des fibroblastes embryonnaires (MEF triple knock out ou TKO) ont ensuite été produits à partir de ces cellules ES. Ces MEF TKO prolifèrent plus vite que des MEF sauvages et sont incapables de s'arrêter à la transition $\mathrm{Gl} / \mathrm{S}$ du cycle cellulaire en réponse à divers signaux ou situations physiologiques conduisant normalement à un tel arrêt (dommages à l'ADN induits par irradiation ou traitement par la doxorubicine, retrait du sérum du basse densité, confluence/ inhibition de contact, surexpression d'inhibiteurs du cycle, sénescence) [1, 2]. Ces caractéristiques sont partagées par la plupart des cellules cancéreuses. Comme elles, les MEF TKO sont immortelles en culture, proliférant indéfiniment sans jamais entrer en sénescence. De même, elles sont capables de proliférer à faible densité et en absence de support (capacité à proliférer en agar mou). Néanmoins, ces travaux montrent également que les cellules TKO ne sont pas totalement transformées puisqu'elles restent incapables de former des tumeurs in vivo lorsqu'elles sont transplantées chez des souris nude athymiques [1, 2]. De fait, la présence d'un second événement oncogénique (dans cette expérience, l'expression d'une forme oncogénique de Ras) reste requise pour la formation de telles tumeurs.

En conclusion, ces deux études montrent que la famille $\mathrm{pRb}$ joue le rôle « d'intégrateur» pour de multiples signaux dont la seule caractéristique commune est de provoquer un arrêt du cycle. Cette fonction ressemble à celle qu'exerce la protéine p53 dans la réponse aux signaux génotoxiques. 
Dans leur ensemble, ces «intégrateurs» constituent les éléments essentiels du système de surveillance de l'état physiologique de la cellule ; un système partiellement ou totalement inactivé dans toutes les cellules cancéreuses.

1. Sage J, Mulligan GJ, H ardy LD, et al. Targeted disruption of the three Rb-related genes leads to loss of $\mathrm{G} 1$ control and immortalization. Genes Dev $2000 ; 14: 3037-50$

2. Dannenberg JH, Van Rossum A, Schuijff L, Te Riele $\mathrm{H}$. Ablation of the retinoblastoma gene family deregulates $\mathrm{G} 1$ control causing immortalization and increased cell turn over under growth restricting conditions. Genes Dev $2000 ; 14$ : 305164.

3. Sardet C, Le Cam L, Fabbrizio E, Vidal M. E2Fs and the Retinoblastoma Protein family. In : Karin M, Ghysdael J, Yaniv M, ed. Progress in Gene Expression 1997, Oncogenes as transcriptional regulators. Birkhauser Verlag publisher, Basel, Swiss ; 2 : 1-62.

4. Mulligan G, Jacks T. 1 The retinoblastoma gene family: cousins with overlapping interests. Trends Genets $998 ; 14: 223-9$.
5. Masciullo V, Khalili K, Giordano A. The Rb family of cell cycle regulatory factors: clinical implications. Int J Oncol $2000 ; 17: 897-902$.

6. Jacks T, Fazeli A, Schmitt EM, Bronson RT, Goodell MA, Weinberg RA. Effects of an Rb mutation in the mouse. Nature $1992 ; 359: 295-300$. 7. Lee EY, Chang CY, Hu N, et al. Mice deficient for $\mathrm{Rb}$ are nonviable and show defects in neurogenesis and haematopoiesis. Nature 1992 ; 359 : 288-94.

8. Herrera RE, Sah VP, Williams BO, Makela TP, Weinberg RA, Tyler J. Altered cell cycle kinetics, gene expression, and $\mathrm{Gl}$ restriction point regulation in RB-deficient fibroblasts. Mol Cell Biol 1996 ; $16: 2402-7$

9. Classon M, Salama S, Gorka C, Mulloy R, Braun $P, H$ arlow E. Combinatorial roles for $P R B$, p107, and p130 in E2F-mediated cell cycle control. Proc Natl Acad Sci USA $2000 ; 97: 10820-5$. 10. Classon M, Kennedy BK, Mulloy R, Harlow E. $O$ pposing roles of $\mathrm{pRB}$ and $\mathrm{p} 107$ in adipocyte differentiation. Proc Natl Acad Sci USA 2000; 97 : 10826-31

11. Cobrinik D, Lee MH, Hannon G, et al. Shared role of the $p R B$-related $p 130$ and $p 107$ proteins in Limb development. Genes Dev 1996 ; 10 : 1633144.

12. Hurford RK, Cobrinik D, Lee MO, Dyson N. $\mathrm{pRb}$ and $\mathrm{p} 107 / \mathrm{p} 130$ are required for the regulated expression of different sets of E2F responsive genes. Genes Dev $1997 ; 11: 1447-63$.
13. Lee MH, Williams BO, Mulligan G, et al. Targeted disruption of p107: functional overlap between p107 and Rb. Genes Dev $1996 ; 10$ : 1621-32. 14. LeCoulter J, Kablar B, Hardy R, et al. Straindependent myeloid hyperplasia, growth deficiency, and accelerated cell cycle in mice lacking the pRb-related p107 gene. Mol Cell Biol 1998; $18: 7455-65$.

15. LeCoulter J, Kablar B, Whyte J, Ying C, Rudnicki MA. Strain-dependent embryonic lethality in mice lacking the retinotoma-related p130 gene. Development $1998 ; 125: 4669-79$.

16. Robanus-Maandag E, Dekker M, van der Valk $M$, et al. p107 is a suppressor of retinoblastoma development in pRb-deficient mice. Genes Dev $1998 ; 12$ : 1599-609.

\section{Éric Fabbrizio \\ Claude Sardet}

Institut de gén étique moléculaire de M ontpellier, Cnrs UMR5535, 1919, route de M ende, 34293 M ontpellier Cedex 5, France.
Un pied à l'étrier pour l'otosclérose. La fonction auditive est de mieux en mieux comprise grâce à l'identification, au cours de ces dernières années, de nombreux gènes impliqués dans des surdités, isolées ou syndromiques. Plus de 90 gènes ont été répertoriés qui interviennent dans le développement ou la fonction de l'oreille interne [1]. Toutefois, on ignore encore tout de l'otosclérose, une maladie entraînant une surdité progressive débutant au cours de la troisième décennie de la vie, très répandue dans le monde occidental puisque sa prévalence est de 0,2 à $1 \%$. Elle est la conséquence d'une sclérose de l'os enchondral de la capsule labyrinthique qui provoque le blocage de la base de l'étrier dans la fenêtre ovale et empêche sa mobilité. A cette surdité de conduction s'ajoute une composante neuro-sensorielle due à des foyers d'otosclérose cochléaire. L'étiologie en est complexe et mal connue. O utre les facteurs environ- nementaux, l'éventualité d'une cause virale, il existe une composante génétique: les études épidémiologiques sont en faveur d'une transmission autosomique à pénétrance réduite. En 1998, I'analyse de liaison d'une grande famille permit de situer un locus en 15q26.1 [2], mais aucun gène n'a encore été localisé jusqu'à présent sur ce locus OTSC 1 . Tout récemment, un deuxième locus (OTSC 2 ) vient d'être trouvé en 7q34-36 grâce à une grande famille belge où l'otosclérose ségrège selon un mode dominant [3]. Dans cet intervalle 7q34$7 q 36$, on trouve deux gènes: $\operatorname{TIF} 1 \alpha$, un co-facteur transcriptionnel nécessaire à l'activité inhibitrice de l'acide rétinoïque (qui pourrait donc intervenir dans la différenciation et le développement de la capsule otique), et surtout le gène codant pour une lysine hydroxylase du procollagène, PLOD3. [4]. On sait que les trois gènes PLOD humains interviennent dans la biosynthèse du col- lagène, et que l'un d'entre eux, PLOD1, est impliqué dans le syndrome d'Ehlers-Danlos de type VI, une maladie du tissus conjonctif. Or, il a été démontré que PLOD3 interagit avec le TNF $\alpha$ (tumor-necrosis factor $\alpha$ ) qui joue un rôle important dans la pathogénie de l'arthrite en contrôlant la dégradation du cartilage. PLOD3 pourrait être impliqué dans l'homéostasie du cartilage de l'oreille moyenne et interne en interférant dans la réponse des chondrocytes aux stimulus transmis par le TNF $\alpha$. II importe donc d'étudier cet excellent candidat dans les cas d'otosclérose dont le locus se situe en 7q34-36.
[1. Steel KP, Kros C]. Nat Genet 2001 ; 27 : 143-9.]
[2. Tomek MS, et al. Hum Molec Genet 1998; $7: 285-90$.]
[3. Van den Bogaert K, et al. Am J H um Gen et $2001 ; 68$ : 495-50.]
[4. Passoja K, et al. Proc Nat Acad Sci USA 1998; 95: 10482-6.] 